\title{
An Energy Regularization for Cauchy Problems of Laplace Equation in Annulus Domain
}

\author{
Houde Han ${ }^{1}$, Leevan Ling ${ }^{2, *}$ and Tomoya Takeuchi ${ }^{3}$ \\ ${ }^{1}$ Department of Mathematical Sciences, Tsinghua University, Beijing 100084, \\ China. \\ ${ }^{2}$ Department of Mathematics, Hong Kong Baptist University, Kowloon Tong, \\ Hong Kong. \\ ${ }^{3}$ Department of Mathematics, North Carolina State University, Raleigh, \\ North Carolina 27695-8212, USA.
}

Received 20 January 2010; Accepted (in revised version) 6 September 2010

Available online 13 October 2010

\begin{abstract}
Detecting corrosion by electrical field can be modeled by a Cauchy problem of Laplace equation in annulus domain under the assumption that the thickness of the pipe is relatively small compared with the radius of the pipe. The interior surface of the pipe is inaccessible and the nondestructive detection is solely based on measurements from the outer layer. The Cauchy problem for an elliptic equation is a typical ill-posed problem whose solution does not depend continuously on the boundary data. In this work, we assume that the measurements are available on the whole outer boundary on an annulus domain. By imposing reasonable assumptions, the theoretical goal here is to derive the stabilities of the Cauchy solutions and an energy regularization method. Relationship between the proposed energy regularization method and the Tikhonov regularization with Morozov principle is also given. A novel numerical algorithm is proposed and numerical examples are given.
\end{abstract}

AMS subject classifications: 65N12, 65N15, 65N21

Key words: Inverse problem, stability, error bounds, Tikhonov regularization, method of fundamental solution.

\section{Introduction}

To provide a safe and reliable mode of energy transport and improve general awareness of the benefits of the pipeline industry, corrosion prevention, preventing outside damage are the high priority tasks. Nevertheless, the recent promotion of high-pressure pipelines

${ }^{*}$ Corresponding author. Email addresses: hhan@math.tsinghua.edu.cn (H. Han), lling@hkbu.edu.hk (L. Ling), tntakeuc@ncsu .edu (T. Takeuchi) 
by different Australian Pipeline Industry Association makes the problems of corrosion detection equally significant. According to the Association of Oil pipe Lines (AOPL), the most recent Crack Detection Tools includes ultrasonic crack detection, magnetic flux leakage, and elastic wave tool. "Some of these technologies are fairly new and still developing," quoted from the AOPL's Pipeline Industry Facts. The wide variety of defects in pipelines can be classified into three major groups: defects due to corrosion, defects generated by mechanical damage, and cracks created by stresses in the pipe wall. Defects of these three groups are mathematically different; each requires specific techniques and models.

If we focus on defects due to corrosion, the inverse problem considered is to nondestructively determine information about the corrosion that occurs on the interior surface of the pipeline. The only accessible data are the electrostatic measurements on the exterior surface of the pipeline. We assume that the thickness of the pipeline is comparatively small with respect to its diameter. This includes the crude oil pipeline: for example, the 2005 El Sharara-Mellitah Onshore Pipeline project of the AGIP Oil Company engaged a $400 \mathrm{~km}$ of pipelines of $762 \mathrm{~mm}$ overall diameter with $11.30 \mathrm{~mm}$ thickness.

Inglese [1] modeled the problem of determining quantitative information about corrosion by Laplace's equation

$$
\triangle u=0, \quad x \in \Omega,
$$

with unknown (interior) boundary conditions. As the thickness of the coating goes to zero, Buttazzo and Kohn [2] observed the arising of mixed boundary conditions. Based on the Faraday's law, corrosion or mass loss is proportional to the normal current flux. The main focuses of this paper are the stability and the numerical algorithm for this inverse problem. Surface with corrosion is usually rough on which a thin coating effect applies. Let $\Gamma_{\text {out }}$ and $\Gamma_{\text {in }}$ be the outer and inner boundary of the annulus domain $\Omega$, respectively. After linearization, one gets

$$
u_{v}(x)+\gamma(x) u(x)=0, \quad x \in \Gamma_{\text {in }}
$$

where $u_{v}$ is the outer normal derivative of $u$ on the boundary. Using the potential model introduced by Ingless and Santosa [3], defects due to corrosion can be described by mixed boundary conditions. In (1.2), $\gamma(x)$ is the coefficient of energy exchange and $\gamma(x) \geq 0$ indicates corrosion damage. The inverse problem here is to find $\gamma(x)$ given knowledge of

$$
u(x)=f(x) \quad \text { and } \quad u_{v}(x)=g(x), \quad x \in \Gamma_{\text {out }} .
$$

Derived from (1.1) and (1.2), the conservation of charge forces

$$
\int_{\Gamma_{\text {out }}} g-\int_{\Gamma_{\text {in }}} \gamma u=0
$$

indicates that the flux through $\Gamma_{\text {out }}$ is not zero. Protter and Weinberger [4] showed that, without loss of generality, choosing $f \geq 0$ ensure $u>0$ in $\bar{\Omega}$ if both $\gamma$ and $f$ have positive Lebesgue measure. The exchange coefficient could be computed by first solving the 
Cauchy problem for Laplace's equations (1.1) and (1.3), and secondly by (1.2) to obtain

$$
\gamma(x)=-\left.\frac{u_{v}}{u}\right|_{\Gamma_{\text {in }}} .
$$

The Cauchy problem for an elliptic equation is a typical ill-posed problem whose solution does not depend continuously on the boundary data. That is, a small error in the specified data may result in an enormous error in the numerical solution. The Cauchy problem for the Laplace equation also arises from many branches of science and engineering such as non-destructive testing [5], steady-state inverse heat conduction [6], and electro-cardiology [7]. Numerical methods for solving the Cauchy problem for Laplace's equation were widely studied; for example, Han et al. [8,9], Falk and Monk [10], Ang, Nghia and Tam [11], Berntsson and Eldén [12], Reinhardt et al. [13], Cheng, Hon, and et al. [14], Hon, Wei and et al. [15, 16], Takeuchi and Yamamoto [17], Leitão [18], Wei, Qin and Shi [19], and etc. Methods specified for corrosion problem include the thin plate approximation by Inglese [1] on plate geometry and the boundary element method by Yang, Choulli and Cheng [20] on pipe.

\section{Stability and regularization in $H^{s+1 / 2}(\Omega)$}

We consider the following Cauchy problem of Laplace equation:

$$
\left\{\begin{array}{l}
\Delta u=0, \quad x \in \Omega, \\
\left.u\right|_{\Gamma_{\text {out }}}=f, \\
\left.\partial_{r} u\right|_{\Gamma_{\text {out }}}=g
\end{array}\right.
$$

where $\Omega \in \mathbb{R}^{2}$ is a bounded domain with boundary $\Gamma_{\text {in }} \cup \Gamma_{\text {out }}$ such that

$$
\Gamma_{\text {in }}=\left\{x:|x|=R_{i}\right\} \quad \text { and } \quad \Gamma_{\text {out }}=\left\{x:|x|=R_{o}\right\}, \quad 0<R_{i}<R_{o} .
$$

Here, $R_{i}$ and $R_{o}$ are the inner and outer radius, respectively.

For the given $f$ and $g$ on $\Gamma_{\text {out }}$, problem (2.1) is an improperly posed problem; namely, the solution of (2.1) is not continuously dependent on the Cauchy data $f$ and $g$.

In practical applications, the given Cauchy data usually contains certain noise. Instead of the exact data $f$ and $g$, we only have

$$
f_{\varepsilon}=f+\text { noise }, \quad \text { and } \quad g_{\varepsilon}=g+\text { noise. }
$$

The exact assumptions imposed on the "noise" will be specified in the next section.

In this paper, we study the continuous dependence of the solution of (2.1) on the Cauchy data provided that the solution of (2.1) satisfying certain restrictions. 


\subsection{Notations: norms and functionals}

The solution of the Cauchy problem, if it exists, is expressed,

$$
u(r, \theta)=a_{0}+b_{0} \log \frac{r}{R_{o}}+\sum_{k=1}^{\infty}\left[\left(a_{k} r^{k} \cos k \theta+b_{k} r^{k} \sin k \theta\right)+\left(c_{k} r^{-k} \cos k \theta+d_{k} r^{-k} \sin k \theta\right)\right],
$$

where $r \in\left[R_{i}, R_{o}\right]$, and $\theta=[0,2 \pi)$. It is straightforward to show that

$$
a_{0}=\frac{1}{2 \pi R_{o}} \int_{\Gamma_{o}} u d \sigma
$$

Let $s$ be a real number. We define the following $\||| \cdot||-$-norm for a $2 \pi$ periodic function, in the form of

$$
h(\theta)=\alpha_{0}+\sum_{k=1}^{\infty}\left(\alpha_{k} \cos k \theta+\beta_{k} \sin k \theta\right)
$$

as

$$
\|h(\theta) \mid\|_{s}^{2}:=\alpha_{0}^{2}+\sum_{k=1}^{\infty} k^{2 s+1}\left(\alpha_{k}^{2}+\beta_{k}^{2}\right) .
$$

Let $r$ be fixed such that $R_{i} \leq r \leq R_{o}$. Consider any closed circular path in $\Omega$

$$
\Gamma_{r}=\{x:|x|=r\} .
$$

For the solution in the form of (2.2), we introduce the following notations:

$$
\begin{aligned}
\left\|\partial_{\theta} u\right\|_{s-1, \Gamma_{r}}^{2} & :=\frac{1}{r}\left\|\partial_{\theta} u_{\mid r}(\theta)\right\|_{s-1}^{2} \\
& =\sum_{k=1}^{\infty} k^{2 s+1}\left[\left(a_{k} r^{k-\frac{1}{2}}+c_{k} r^{-k-\frac{1}{2}}\right)^{2}+\left(b_{k} r^{k-\frac{1}{2}}+d_{k} r^{-k-\frac{1}{2}}\right)^{2}\right], \\
\left\|\partial_{r} u\right\|_{s-1, \Gamma_{r}}^{2} & :=\left.r\left\|\partial_{r} u_{\left.\right|_{r}}(\theta)\right\|\right|_{s-1} ^{2} \\
& =\frac{b_{0}^{2}}{r}+\sum_{k=1}^{\infty} k^{2 s+1}\left[\left(a_{k} r^{k-\frac{1}{2}}-c_{k} r^{-k-\frac{1}{2}}\right)^{2}+\left(b_{k} r^{k-\frac{1}{2}}-d_{k} r^{-k-\frac{1}{2}}\right)^{2}\right] .
\end{aligned}
$$

For the sake of simpler notations, we also define a semi-norm by

$$
|u|_{s, \Gamma_{r}}^{2}:=\frac{1}{r}\left(a_{o}^{2}+\|\| \partial_{\theta} u_{\left.\right|_{r}}(\theta)||_{s-1}^{2}\right)=\frac{a_{0}^{2}}{r}+\left\|\partial_{\theta} u\right\|_{s-1, \Gamma_{r}}^{2} .
$$

We now define a functional $J_{s}$ for the $u$ in (2.2) as

$$
J_{s}(r)(u)=\frac{a_{0}^{2}}{r}+\left\|\partial_{r} u\right\|_{s-1, \Gamma_{r}}^{2}+\left\|\partial_{\theta} u\right\|_{s-1, \Gamma_{r}}^{2}=|u|_{s, \Gamma_{r}}^{2}+\left\|\partial_{\theta} u\right\|_{s-1, \Gamma_{r}}^{2} .
$$

Substituting (2.4a) and (2.4b) into (2.6), we arrive at

$$
J_{s}(r)(u)=\frac{a_{0}^{2}+b_{0}^{2}}{r}+2 \sum_{k=1}^{\infty} k^{2 s+1}\left[\left(a_{k}^{2}+b_{k}^{2}\right) r^{2 k-1}+\left(c_{k}^{2}+d_{k}^{2}\right) r^{-2 k-1}\right] .
$$


Proposition 2.1. The semi-norm $|u|_{s, \Gamma_{r}}^{2}$ for all Cauchy solutions (2.1) becomes a norm when $r=R_{o}$; namely,

$$
|u|_{s, \Gamma_{R_{o}}}^{2}=\frac{1}{R_{o}}\left\|u\left(R_{o}, \theta\right)\right\|_{s}^{2}
$$

Let $\|u\|_{s+1 / 2, \Omega}$ be an equivalent norm to that in $H^{s+1 / 2}(\Omega)$ defined by

$$
\|u\|_{S+\frac{1}{2}, \Omega}^{2}:=\int_{R_{i}}^{R_{o}} J_{\mathcal{S}}(r)(u) d r .
$$

Furthermore, for $s=1 / 2$, a direct computation yields that

$$
\|u\|_{1, \Omega}^{2}:=\int_{R_{i}}^{R_{o}} J_{\frac{1}{2}}(r)(u) d r=\frac{\left(R_{o}-R_{i}\right)}{R_{o}} a_{0}^{2}+\int_{\Omega} \nabla u \cdot \nabla u d x .
$$

For the solution in the form of (2.2), we know that $\left(\int_{R_{i}}^{R_{o}} J_{1 / 2}(r)(u) d r\right)^{1 / 2}$ is an equivalent norm of $u$ in $H^{1}(\Omega)$.

Throughout this paper, we use $J(r)$ to abbreviate $J_{s}(r)(u)$ when no confusion arises.

\subsection{Three-line inequality}

A three-line inequality was derived in [8] to analysis the Cauchy on rectangular domains that have two insulated vertical boundaries, $\Gamma_{0}$ and $\Gamma_{1}$ are respectively the top and bottom boundaries. In this section, we will derive an analogue for our problem on annulus domains. For any $r_{1}$ and $r_{2}$ with $R_{i} \leq r_{1}<r_{2} \leq R_{o}$, let $\sqrt{r_{1} r_{2}}$ be the geometric mean of $r_{1}$ and $r_{2}$. Then, we have

$$
J\left(\sqrt{r_{1} r_{2}}\right)=\frac{a_{0}^{2}+b_{0}^{2}}{\sqrt{r_{1} r_{2}}}+2 \sum_{k=1}^{\infty} k^{2 s}\left[\left(a_{k}^{2}+b_{k}^{2}\right) r_{1}^{k-\frac{1}{2}} r_{2}^{k-\frac{1}{2}}+\left(c_{k}^{2}+d_{k}^{2}\right) r_{1}^{-k-\frac{1}{2}} r_{2}^{-k-\frac{1}{2}}\right] .
$$

By the Cauchy-Schwarz inequality, we have

$$
J\left(\sqrt{r_{1} r_{2}}\right) \leq J\left(r_{1}\right)^{\frac{1}{2}} J\left(r_{2}\right)^{\frac{1}{2}} .
$$

Consider any $r \in\left[R_{i}, R_{o}\right]$. We define

$$
\gamma(r)=\frac{\log r-\log R_{i}}{\log R_{o}-\log R_{i}}
$$

and write $r=R_{i}^{1-\gamma(r)} R_{o}^{\gamma(r)}$.

It is easy to show that $0 \leq \gamma(r) \leq 1$ for all $r \in\left[R_{i}, R_{0}\right]$. Hence, we can express $\gamma(r)$ as

$$
\gamma(r)=\sum_{k=1}^{\infty} \frac{\ell_{k}}{2^{k^{\prime}}}
$$


where $\ell_{k} \in\{0,1\}$ for all $k$. We define a convergent sequence based on (2.11),

$$
\gamma_{j}(r)=\sum_{k=1}^{j} \frac{\ell_{k}}{2^{k}} \rightarrow \gamma(r), \quad \text { as } j \rightarrow \infty .
$$

Immediately, we have

$$
R_{i}^{1-\gamma_{j}(r)} R_{o}^{\gamma_{j}(r)} \rightarrow R_{i}^{1-\gamma(r)} R_{o}^{\gamma(r)}=r, \quad \text { as } j \rightarrow \infty .
$$

Using the estimate given in (2.9), we also have

$$
J\left(R_{i}^{1-\gamma_{j}(r)} R_{o}^{\gamma_{j}(r)}\right) \leq J\left(R_{i}\right)^{1-\gamma_{j}(r)} J\left(R_{o}\right)^{\gamma_{j}(r)} .
$$

Letting $j \rightarrow \infty$, and by (2.12) and (2.13), we obtain a three-line inequality

$$
J(r) \leq J\left(R_{i}\right)^{1-\gamma(r)} J\left(R_{o}\right)^{\gamma(r)}, \text { for } R_{i} \leq r \leq R_{o},
$$

with $\gamma(r)$ given in (2.10).

\subsection{Continuous dependency on Cauchy data}

Suppose the solution $u$ of problem (2.1) satisfies the following assumption

$$
J\left(R_{i}\right)=|u|_{s, \Gamma_{R_{i}}}^{2}+\left\|\partial_{r} u\right\|_{s-1, \Gamma_{R_{i}}}^{2} \leq M<\infty,
$$

where $M$ is a positive constant. Moreover, we assume that there is another positive constant $\varepsilon<M R_{i} / R_{0}$, such that

$$
J\left(R_{o}\right)=|u|_{s, \Gamma_{R_{o}}}^{2}+\left\|\partial_{r} u\right\|_{S-1, \Gamma_{R_{o}}}^{2} \leq \varepsilon
$$

Using the three-line inequality (2.14) and under the assumptions (2.15) and (2.16), we obtain a bound

$$
\|u\|_{S+\frac{1}{2}, \Omega}^{2} \leq \int_{R_{i}}^{R_{o}} J\left(R_{i}\right)^{1-\gamma(r)} J\left(R_{o}\right)^{\gamma(r)} d r \leq M \int_{R_{i}}^{R_{o}}\left(\frac{\varepsilon}{M}\right)^{\gamma(r)} d r .
$$

Using (2.10), the integrand of (2.17) can be expressed as

$$
\left(\frac{\varepsilon}{M}\right)^{\gamma(r)}=\left(\frac{\varepsilon}{M}\right)^{\frac{\log \left(r / R_{i}\right)}{\log \left(R_{0} / R_{i}\right)}}=\lambda^{\log \left(\frac{r}{R_{i}}\right)}=\left(\frac{r}{R_{i}}\right)^{\log \lambda},
$$

with

$$
\lambda:=\left(\frac{\varepsilon}{M}\right)^{\left(\log \frac{R_{0}}{R_{i}}\right)^{-1}} .
$$


By the imposed condition $\varepsilon<M R_{i} / R_{o}$, we have

$$
-\log \lambda=\frac{\log (M / \varepsilon)}{\log \left(R_{o} / R_{i}\right)}>1 .
$$

Therefore, the integral in (2.17) can be bounded by

$$
\begin{aligned}
\int_{R_{i}}^{R_{o}}\left(\frac{\varepsilon}{M}\right)^{\gamma(r)} d r & =\int_{R_{i}}^{R_{o}}\left(\frac{r}{R_{i}}\right)^{\log \lambda} d r=R_{i} \int_{1}^{\frac{R_{o}}{R_{i}}}(\xi)^{\log \lambda} d \xi \\
& =R_{i} \frac{\left(R_{o} / R_{i}\right)^{1+\log \lambda}-1}{1+\log \lambda}=\frac{R_{i}-R_{o}\left(R_{i} / R_{o}\right)^{-\log \lambda}}{-\log \lambda-1} \\
& \leq \frac{R_{i}}{-\log \lambda-1} .
\end{aligned}
$$

Therefore, (2.17) implies that the Cauchy problem (2.1) depends continuously on the Cauchy data. We conclude the stability result in the following lemma.

Lemma 2.1. Suppose that the solution $u$ of the Cauchy problem (2.1) satisfies the restriction (2.15) for a constant $0<M<\infty$ and (2.16) for a constant $\varepsilon<M R_{i} / R_{o}$, then $\|u\|_{s+1 / 2, \Omega}^{2}$ defined as (2.8) depends continuously on the Cauchy data $f$ and $g$ on $\Gamma_{\text {out }}$, namely

$$
\|u\|_{s+\frac{1}{2}, \Omega}^{2} \leq M R_{i} \log \frac{R_{o}}{R_{i}}\left(\log \frac{R_{i}}{R_{o}} \frac{M}{\varepsilon}\right)^{-1},
$$

where $C_{0}$ is a positive constant depending on $R_{i}$ and $R_{o}$.

Proof. We have shown that

$$
\|u\|_{s+\frac{1}{2}, \Omega}^{2} \leq \frac{M R_{i}}{-\log \lambda-1}=\frac{M R_{i}}{\left(\frac{\log (M / \varepsilon)}{\log \left(R_{o} / R_{i}\right)}\right)-1} .
$$

An important feature of the proven bound is that all terms on the right hand side can be explicitly evaluated.

\subsection{Energy regularization}

The energy regularization method is recently applied for image deblurring [21]. In our case, using Lemma 2.1, the Cauchy problem is well-posed in a subspace of $H^{s+1 / 2}(\Omega)$. In particular, the subspace is given by

$$
U_{\varepsilon}:=\left\{u \in H^{s+\frac{1}{2}}(\Omega): \Delta u=0, \text { in } \Omega \text {, and }\left|u-f_{\varepsilon}\right|_{s, \Gamma_{R_{o}}}^{2}+\left\|\partial_{\theta} u-g_{\varepsilon}\right\|_{s-1, \Gamma_{R_{o}}}^{2} \leq \varepsilon\right\} .
$$

Now we define our regularized solution through a minimization

$$
u_{\varepsilon}:=\arg \min _{w \in U_{\varepsilon}} J\left(R_{i}\right)(w) .
$$


Theorem 2.1. Suppose that the solution $u^{*}$ of the Cauchy problem (2.1) satisfies the assumption

$$
J\left(R_{i}\right)\left(u^{*}\right) \leq M<\infty,
$$

then the regularized solution defined by (2.18) converges to the exact solution as the noise level $\varepsilon \rightarrow 0$,

$$
\left\|u_{\varepsilon}-u^{*}\right\|_{s+\frac{1}{2}, \Omega}^{2} \leq M R_{i} \log \frac{R_{o}}{R_{i}}\left(\log \frac{R_{i}}{R_{o}} \frac{M}{\varepsilon}\right)^{-1} .
$$

Proof. Note that $J\left(R_{i}\right)\left(u_{\varepsilon}-u^{*}\right) \leq 2 J\left(R_{i}\right)\left(u^{*}\right) \leq 2 M$ and that

$$
\begin{aligned}
J\left(R_{o}\right)\left(u_{\varepsilon}-u^{*}\right) & =\left|u_{\varepsilon}-f\right|_{s, \Gamma_{R_{o}}}^{2}+\left\|\partial_{r} u_{\varepsilon}-g\right\|_{s-1, \Gamma_{R_{o}}}^{2} \\
& \leq\left|u_{\varepsilon}-f_{\varepsilon}\right|_{s, \Gamma_{R_{o}}}^{2}+\left\|\partial_{r} u_{\varepsilon}-g_{\varepsilon}\right\|_{s-1, \Gamma_{R_{o}}}^{2}+\left|f_{\varepsilon}-f\right|_{s, \Gamma_{R_{o}}}^{2}+\left\|g_{\varepsilon}-g\right\|_{s-1, \Gamma_{R_{o}}}^{2} \\
& \leq 2 \varepsilon
\end{aligned}
$$

Thus, applying Lemma 2.1 to $u_{\varepsilon}-u^{*}$ proves the theorem.

\subsection{Relation with the Tikhonov regularization with Morozov principle}

This section studies the relationship between our regularization method and the Tikhonov regularization with Morozov discrepancy principle. For Tikhonov regularization, we refer to Baumeister [22], Engl, Hanke and Neubauer [23], Groetsch [24, 25], Hofmann [26, 27], Vasin [28] and references therein.

We make a natural assumption on the Cauchy data and the noise level, that is we assume that

$$
\left|f_{\varepsilon}\right|_{s, \Gamma_{R_{o}}}^{2}+\left\|g_{\varepsilon}\right\|_{s-1, \Gamma_{R_{o}}}^{2}>\varepsilon
$$

It is easy to see that the assumption guarantees that the existence of nontrivial solution $u_{\varepsilon}$ for (2.18). Moreover, it is easy to see that the above assumption grantees that the solution $u_{\varepsilon}$ attains the minimum on

$$
\left|u_{\varepsilon}-f_{\varepsilon}\right|_{s, \Gamma_{R_{o}}}^{2}+\left\|\partial_{r} u_{\varepsilon}-g_{\varepsilon}\right\|_{s-1, \Gamma_{R_{o}}}^{2}=\varepsilon .
$$

Let $\alpha$ be a positive number and $u_{\alpha}^{\varepsilon}$ be the minimization solution of the following Tikhonov functional

$$
u_{\alpha}^{\varepsilon}:=\arg \min _{\Delta u=0}\left(\left|u-f_{\varepsilon}\right|_{s, \Gamma_{R_{o}}}^{2}+\left\|\partial_{r} u-g_{\varepsilon}\right\|_{s-1, \Gamma_{R_{o}}}^{2}+\alpha J\left(R_{i}\right)(u)\right) .
$$

It is well known that there exists $\alpha(\varepsilon)>0$ for the Mirozov principle such that

$$
\left|u_{\alpha(\varepsilon)}^{\varepsilon}-f_{\varepsilon}\right|_{s, \Gamma_{R_{o}}}^{2}+\left\|\partial_{r} u_{\alpha(\varepsilon)}^{\varepsilon}-g_{\varepsilon}\right\|_{s-1, \Gamma_{R_{o}}}^{2}=\varepsilon
$$


The definition of $u_{\alpha(\varepsilon)}^{\varepsilon}$ yields

$$
\begin{aligned}
& \left|u_{\alpha(\varepsilon)}^{\varepsilon}-f_{\varepsilon}\right|_{s, \Gamma_{R_{o}}}^{2}+\left\|\partial_{r} u_{\alpha(\varepsilon)}^{\varepsilon}-g_{\varepsilon}\right\|_{s-1, \Gamma_{R_{o}}}^{2}+\alpha(\varepsilon) J\left(R_{i}\right)\left(u_{\alpha(\varepsilon)}^{\varepsilon}\right) \\
\leq & \left|u^{\varepsilon}-f_{\varepsilon}\right|_{s, \Gamma_{R_{o}}}^{2}+\left\|\partial_{r} u^{\varepsilon}-g_{\varepsilon}\right\|_{s-1, \Gamma_{R_{o}}}^{2}+\alpha(\varepsilon) J\left(R_{i}\right)\left(u^{\varepsilon}\right),
\end{aligned}
$$

and hence $J\left(R_{i}\right)\left(u_{\alpha(\varepsilon)}^{\varepsilon}\right) \leq J\left(R_{i}\right)\left(u^{\varepsilon}\right)$. On the other hand, $J\left(R_{i}\right)\left(u_{\alpha(\varepsilon)}^{\varepsilon}\right) \geq J\left(R_{i}\right)\left(u^{\varepsilon}\right)$, by the minimization property of $u^{\varepsilon}$. We have

$$
J\left(R_{i}\right)\left(u_{\alpha(\varepsilon)}^{\varepsilon}\right)=J\left(R_{i}\right)\left(u^{\varepsilon}\right) .
$$

In other words, the solutions obtained by the energy regularization and by the Tikhonov regularization with Morozov principle will have the same energy on the inner boundary. Note that the convex functional $J$ takes the unique minimum over the constraints, and thus $J\left(R_{i}\right)\left(u_{\alpha(\varepsilon)}^{\varepsilon}\right)=J\left(R_{i}\right)\left(u^{\varepsilon}\right)$ means $u_{\alpha(\varepsilon)}^{\varepsilon}=u^{\varepsilon}$.

The representation (2.2) of the solution of the Cauchy problem eliminates the condition $\Delta u=0$ from the constraints of both the minimization problems (2.18) and (2.20), and the problems become the minimization problems on the coefficients $\left\{a_{0}, b_{0}, a_{k}, b_{k}, c_{k}, d_{k}\right\}_{k=1}^{\infty}$. However, the problem (2.18) still has the constraint on the Cauchy data,

$$
\left|u^{\varepsilon}-f_{\varepsilon}\right|_{s, \Gamma_{R_{o}}}^{2}+\left\|\partial_{r} u^{\varepsilon}-g_{\varepsilon}\right\|_{s-1, \Gamma_{R_{o}}}^{2} \leq \varepsilon .
$$

On the other hand there is no constraint in the problem (2.20), which makes the numerical computation easier. Finding the regularization parameter $\alpha$ satisfying the Morozov principle seems to require us to solve the problem (2.20) with a wide range of $\alpha$, however, effective methods are available to find the parameter with less computation. We refer to Ito and Kunisch [29], Kunisch and Zou [30], Xie and Zou [31].

Once we obtain the coefficients $\left\{a_{0}, b_{0}, a_{k}, b_{k}, c_{k}, d_{k}\right\}_{k=1}^{\infty}$, the regularized solution is represented as (2.2). However, the error in the coefficients are amplified by the factor $r^{k}$ and/or $r^{-k}$, and the numerical solution will have large oscillation. One can introduce the cut off frequency $N_{c}$ and define a solution with the coefficients with $k \leq N_{c}$. The error estimate between exact solution and such a solution can be found in Berntsson and Eldén [12]. Yet, in the next section, we present a different approach based on the method of fundamental solution.

\section{Numerical approach}

\subsection{Implementation with MFS}

We assume that we have the Cauchy data of the form

$$
\begin{aligned}
& f_{\mathcal{\varepsilon}}^{N}(\theta)=\sum_{k=0}^{N} c\left(f_{\mathcal{\varepsilon}}^{N}\right)_{k} \cos k \theta+\sum_{k=1}^{N} s\left(f_{\mathcal{\varepsilon}}^{N}\right)_{k} \sin k \theta, \\
& g_{\mathcal{E}}^{N}(\theta)=\sum_{k=0}^{N} c\left(g_{\mathcal{\varepsilon}}^{N}\right)_{k} \cos k \theta+\sum_{k=1}^{N} s\left(g_{\mathcal{\varepsilon}}^{N}\right)_{k} \sin k \theta,
\end{aligned}
$$


with $\left|u-f_{\varepsilon}\right|_{s, \Gamma_{R_{o}}}^{2}+\left\|\partial_{r} u-g_{\varepsilon}\right\|_{s-1, \Gamma_{R_{o}}}^{2} \leq \varepsilon$, where $u$ is an exact solution we give for a numerical test, and $\left\{c(f)_{k}\right\}_{k=0}^{N}$ and $\left\{s(f)_{k}\right\}_{k=1}^{N}$ are the Fourier coefficients of a function $f$.

We present a numerical procedure for solving a Cauchy problem using the method of fundamental solution (MFS). In literature, the fundamental solutions and Green's functions appear to be some attractive alternatives for solving direct $[32,33]$ and inverse problems $[16,17,34-37]$.

Denote $\Phi(x, \xi)$ to be the fundamental solution of the Laplacian operator. When the source points $\xi$ are located outside the domain $\bar{\Omega}$, the fundamental solution satisfies the Laplace equation in domain $\Omega$. The basic idea of the MFS is to approximate the solution in term of a series of fundamental solutions. By construction, any numerical solution automatically satisfies the differential equation.

Let $\Xi:=\left\{\tilde{\zeta}_{i}\right\}_{i=1}^{m}$ be a set of source points in $\mathbb{R}^{2} \backslash \bar{\Omega}$. We represent the numerical solution by the linear sum of fundamental solution with singularity outside of our domain

$$
u^{N}=\sum_{j=1}^{m} \lambda_{j} \Phi\left(\cdot-\xi_{j}\right)
$$

We determine $\left\{\lambda_{j}\right\}_{j=1}^{m}$ by applying our regularization method, which requires to evaluate the norms $\left|u^{N}-f_{\mathcal{\varepsilon}}^{N}\right|_{s, \Gamma_{R_{o}}}^{2}+\left\|\partial_{r} u^{N}-g_{\mathcal{\varepsilon}}^{N}\right\|_{s-1, \Gamma_{R_{o}}}^{2}$ and the energy

$$
J\left(R_{i}\right)\left(u^{N}\right)=\left|u^{N}\right|_{s, \Gamma_{R_{i}}}^{2}+\left\|\partial_{r} u^{N}\right\|_{s-1, \Gamma_{R_{i}}}^{2}
$$

We expand the trace of $\Phi\left(\cdot-\xi_{j}\right)$ and its normal derivative $\partial_{r} \Phi\left(\cdot-\xi_{j}\right)$ on $\Gamma_{R_{o}}$ in Fourier series

$$
\begin{aligned}
& \left.\Phi\left(\cdot-\xi_{j}\right)\right|_{\Gamma_{R_{o}}}(\theta)=\sum_{k=0}^{N_{f}} A_{k}^{j} \cos k \theta+\sum_{k=1}^{N_{f}} B_{k}^{j} \sin k \theta, \\
& \left.\partial_{r} \Phi\left(\cdot-\xi_{j}\right)\right|_{\Gamma_{R_{o}}}(\theta)=\sum_{k=0}^{N_{f}} C_{k}^{j} \cos k \theta+\sum_{k=1}^{N_{f}} D_{k}^{j} \sin k \theta,
\end{aligned}
$$

for $j=1, \cdots, m$. Here $N_{f}$ is sufficiently large number. Then

$$
\begin{aligned}
& \sum_{j=1}^{m} \lambda_{j} \Phi\left(\cdot-\xi_{j}\right)=\sum_{k=0}^{N_{f}}\left(\sum_{j=1}^{m} A_{k}^{j} \lambda_{j}\right) \cos k \theta+\sum_{k=1}^{N_{f}}\left(\sum_{j=1}^{m} B_{k}^{j} \lambda_{j}\right) \sin k \theta, \\
& \sum_{j=1}^{m} \lambda_{j} \partial_{r} \Phi\left(\cdot-\xi_{j}\right)=\sum_{k=0}^{N_{f}}\left(\sum_{j=1}^{m} C_{k}^{j} \lambda_{j}\right) \cos k \theta+\sum_{k=1}^{N_{f}}\left(\sum_{j=1}^{m} D_{k}^{j} \lambda_{j}\right) \sin k \theta .
\end{aligned}
$$

Thus, $\left|u^{N}-f_{\mathcal{\varepsilon}}^{N}\right|_{s, \Gamma_{R_{o}}}^{2}+\left\|\partial_{r} u^{N}-g_{\mathcal{\varepsilon}}^{N}\right\|_{s-1, \Gamma_{R_{o}}}^{2}$ is written as

$$
\begin{aligned}
\|G \lambda-y\|_{\mathbb{R}^{m}}^{2}:= & \sum_{k=0}^{N_{f}} k^{2 s}\left(\sum_{j=1}^{m} A_{k}^{j} \lambda_{j}-c\left(f_{\varepsilon}^{N}\right)_{k}\right)^{2}+\sum_{k=1}^{N_{f}} k^{2 s}\left(\sum_{j=1}^{m} B_{k}^{j} \lambda_{j}-s\left(f_{\varepsilon}^{N}\right)_{k}\right)^{2} \\
& +R_{o}^{2} \sum_{k=0}^{N_{f}} k^{2(s-1)}\left(\sum_{j=1}^{m} C_{k}^{j} \lambda_{j}-c\left(g_{\varepsilon}^{N}\right)_{k}\right)^{2}+R_{o}^{2} \sum_{k=1}^{N_{f}} k^{2(s-1)}\left(\sum_{j=1}^{m} D_{k}^{j} \lambda_{j}-s\left(g_{\varepsilon}^{N}\right)_{k}\right)^{2},
\end{aligned}
$$


with suitably defined $G$ and $y$. Since in general $N_{f}$ is a sufficiently large number $(N \ll$ $N_{f}$ ), we extend, for instance, the vector $\left\{c\left(f_{\mathcal{\varepsilon}}^{N}\right)\right\}_{k=0}^{N}$ to the vector with $N_{f}$ components by appending 0 to $\left\{c\left(f_{\mathcal{\varepsilon}}^{N}\right)\right\}_{k=0}^{N}$. Similarly, the energy is written as

$$
J\left(u^{N}\right)=\|F \lambda\|_{\mathbb{R}^{m}}^{2},
$$

where $F$ is constructed by using the Fourier coefficients of $\left.\Phi\left(\cdot-\xi_{j}\right)\right|_{\Gamma_{i}}(\theta)$ and $\partial_{r} \Phi(\cdot-$ $\left.\xi_{j}\right)\left.\right|_{\Gamma_{i}}(\theta)$. These allow us to solve (2.18) numerically.

\subsection{Numerical examples}

In this section, we verify the numerical accuracy of the proposed method for the Cauchy problem (2.1). We choose the following function as a test example:

\section{Example 3.1.}

$$
u(r, \theta)=3+r \cos \theta-r^{-2} \cos 2 \theta .
$$

We use $u$ to denote an exact solution instead of using $u^{*}$. The radii of the annulus are fixed $R_{i}=0.8$ and $R_{o}=1$ for both cases. For MFS, we distribute one hundred source points on a circle $\{x:|x|=0.55\}$ and another one hundred source points on a bigger circle $\{x:|x|=1.5\}$. Firstly, we verify the numerical accuracy of the method by observing the error estimate (2.19) against numerical solutions for a fixed $s$. For a given $\varepsilon>0$, the Cauchy data $\left\{f_{\varepsilon}(\theta), g_{\varepsilon}(\theta)\right\}$ is generated as

$$
f_{\varepsilon}(\theta)=u\left(R_{o}, \theta\right)+\sum_{k=0}^{N} \epsilon_{k}^{f} \cos k \theta, \quad \text { and } \quad g_{\varepsilon}(\theta)=\partial_{r} u\left(R_{o}, \theta\right)+\sum_{k=0}^{N} \epsilon_{k}^{g} \cos k \theta,
$$

so that

$$
\varepsilon=\left|u-f_{\varepsilon}\right|_{s, \Gamma_{R_{o}}}^{2}+\left\|\partial_{r} u-g_{\varepsilon}\right\|_{s-1, \Gamma_{R_{o}}}^{2}
$$

where $\left\{\epsilon_{k}^{f}, \epsilon_{k}^{g}\right\}$ are random numbers. We solve the minimization problem (2.18) to compute $\left\|u-u^{N}\right\|_{s+1 / 2, \Omega}^{2}$. Table 1 shows that the asymptotic behavior of the numerical error for $s=0.5$. The authors observe similar tends for all $s \in(-1,1)$; e.g., the numerical solution converges to the exact solution as $\varepsilon \rightarrow 0$.

Suppose two Cauchy data $\left\{f_{\varepsilon}^{1}(\theta), g_{\varepsilon}^{1}(\theta)\right\}$ and $\left\{f_{\varepsilon}^{2}(\theta), g_{\varepsilon}^{2}(\theta)\right\}$ have the same error level

$$
\left|u-f_{\varepsilon}^{1}\right|_{s_{1}, \Gamma_{R_{o}}}^{2}+\left\|\partial_{r} u-g_{\varepsilon}^{1}\right\|_{s_{1}-1, \Gamma_{R_{o}}}^{2}=\left|u-f_{\varepsilon}^{2}\right|_{s_{2}, \Gamma_{R_{o}}}^{2}+\left\|\partial_{r} u-g_{\varepsilon}^{2}\right\|_{s_{2}-1, \Gamma_{R_{o}}{ }^{\prime}}^{2}
$$

for regularization orders $s_{1}<s_{2}$. Although the noise level $\varepsilon$ are equal in both cases, the overall shapes of these Cauchy data are different. The Cauchy data for smaller $s_{1}$ contains rougher noise, whereas the Cauchy data for larger $s_{2}$ contains smoother noise and is closer to the exact Cauchy data. Hence it is maneless to compare the two numerical solutions obtained with these Cauchy data with identical noise level $\varepsilon$. However, it seems interesting to compare numerical solutions obtained with different regularization order $s$ in (2.18) with a given Cauchy data. Therefore, instead of showing the behavior of the error against noise level $\varepsilon$, we investigate the effect of the regularization order $s$ to the numerical solution for a given Cauchy data with the same relative noise level. 
Table 1: Behavior of the numerical error with increasing numbers of Cauchy data.

\begin{tabular}{||c|c|c|c|c|c|c||}
\hline Error $\backslash N$ & $2^{5}$ & $2^{6}$ & $2^{7}$ & $2^{8}$ & $2^{9}$ & $2^{10}$ \\
\hline Abs. & $1.0736 \mathrm{e}-1$ & $8.1313 \mathrm{e}-2$ & $4.3439 \mathrm{e}-2$ & $2.5039 \mathrm{e}-2$ & $1.3907 \mathrm{e}-2$ & $8.6993 \mathrm{e}-3$ \\
Rel. & $2.1067 \mathrm{e}-2$ & $1.5956 \mathrm{e}-2$ & $8.5238 \mathrm{e}-3$ & $4.9133 \mathrm{e}-3$ & $2.7289 \mathrm{e}-3$ & $1.7070 \mathrm{e}-3$ \\
\hline
\end{tabular}

\subsubsection{The effect of choosing $s$ for a given Cauchy data}

This section studies the effect of the regularization order $s$ to the numerical solution with a given Cauchy data.

Our Cauchy data are generated as follows: we fix $2^{8}$ points $\theta_{k}=\pi k 2^{-7}$, for $k=$ $0, \cdots,\left(2^{8}-1\right)$ on $\Gamma_{R_{o}}$ and sample $u$ at these points. Then the discrete noisy Dirichlet data $\mathbf{f}_{\text {noise }} \in \mathbb{R}^{2^{8}}$ is generated by adding random numbers to the exact data $\mathbf{u}=\left\{u\left(R_{0}, \theta_{k}\right)\right\}_{k=0}^{2^{8}-1}$,

$$
\mathbf{f}_{\text {noise }}(k)=u\left(R_{0}, \theta_{k}\right)+\sigma \cdot \operatorname{rand}(k)\left(\max _{\theta \in[0,2 \pi]} u\left(R_{0}, \theta\right)-\min _{\theta \in[0,2 \pi]} u\left(R_{0}, \theta\right)\right),
$$

where $\operatorname{rand}(k) \in[-1,1]$ is random number and $\sigma$ is the prescribed the relative noise level (independent of regularization order $s$ ). We take the Discrete Fourier transform of $\mathbf{f}_{\text {noise }}$ to obtain $f_{\varepsilon}(\theta)$ of the form (3.1a). The Neumann data $g_{\varepsilon}(\theta)$ in (3.1b) is given in the same way. We get the noise level $\varepsilon$ depending on $\sigma$ and $s$ by

$$
\varepsilon(\sigma, s):=\left|u-f_{\varepsilon}\right|_{s, \Gamma_{R_{o}}}^{2}+\left\|\partial_{r} u-g_{\varepsilon}\right\|_{s-1, \Gamma_{R_{o}}}^{2}
$$

for each Cauchy data. In practical situation, since the exact solution is unknown, the noise level $\varepsilon(\sigma, s)$ cannot be obtained in this way, and it is what we must estimate in some way. Our propose here is to verify the performance of the proposed regularization. The exact value of $\varepsilon$ given by (3.2) is used in the computation. If it is not the case, we have to estimate $\varepsilon$. If (the estimated) $\varepsilon$ is too large, our minimization problem, roughly speaking, becomes an unconstrained minimization problem. That is, we ignore the Cauchy data (correspond to $\alpha$ being too big in the Tikhonov regularization). Whereas, if $\varepsilon$ is chosen to small, the constraint is almost the same as $u=f, \partial_{r} u=g$, which means we solve the Cauchy problem without regularization (correspond to $\alpha \simeq 0$ ). If a priori knowledge on exact noise level is assumed to be known; in this case, we can clearly see the effect of the regularization order $s$ to the numerical solution.

It is obvious that $\varepsilon(\sigma) \downarrow 0$ as $\sigma \downarrow 0$. We obtain the numerical solution by solving (2.18) with using the noise level $\varepsilon(\sigma, s)$ for $U_{\varepsilon}$. Henceforth, we denote the numerical solution by $u_{\sigma}^{s}(r, \theta)$ to indicate the relative noise level in the Cauchy data $\sigma$ and the regularization order $s$ of the Sobolev space where the numerical solution lies.

For the numerical error estimations, we compute the relative error of $u_{\sigma}^{\mathcal{S}}(r, \theta)$ over the whole domain $\Omega$ :

$$
E(\sigma, s):=\frac{\left\|u-u_{\sigma}^{s}\right\|_{s+\frac{1}{2}, \Omega}^{2}}{\|u\|_{s+\frac{1}{2}, \Omega}^{2}}
$$




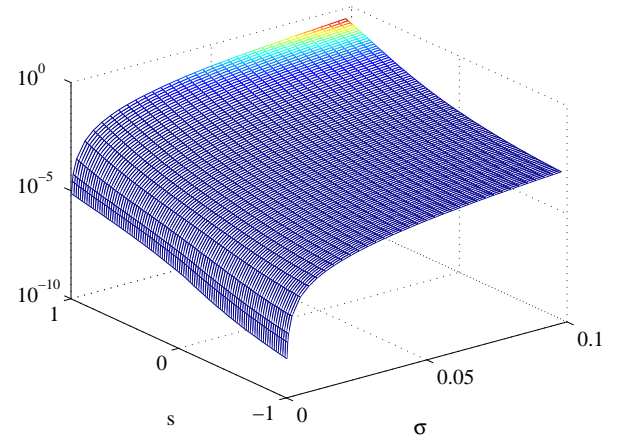

Figure 1: $E(\sigma, s)$ of Example 3.1: Relative error in the numerical solutions in $H^{s+1 / 2}(\Omega)$.

Fig. 1 shows the graph of $E(\sigma, s)$ for $(\sigma, s) \in(0,10 \%] \times[-1,1]$. One can see that the relative error $E$ monotonically decreases as $\sigma \downarrow 0$ and for any fixed $s$ as indicated in (2.19). The author observed that, for relative noise level $\sigma=0$ (i.e., the noise free case), $E(0, s)$ is about machine accuracy $\simeq 10^{-16}$ independent of $s$. Hence, data for $E(0, s)$ is not included in the Fig. 1. Moreover, we observe that $E(\sigma,-1)<E(\sigma, s)$ for all tested $\sigma$ and $s$, but this does not necessarily indicate that the numerical solution of the case $s=-1$ is most accurate among others (since $E$ depends on $s$ ).

For the comparison of the effect of $s$ to the numerical solutions, we plot three numerical solutions on the inner boundary $u_{\sigma}^{s}\left(R_{i}, \theta\right)=u_{5 \%}^{-1}\left(R_{i}, \theta\right), u_{5 \%}^{0.5}\left(R_{i}, \theta\right), u_{5 \%}^{1}\left(R_{i}, \theta\right)$ and the exact solution $u\left(R_{i}, \theta\right)$ in Fig. 2. We authors observe that the results of $s=0$ and $s=-1$ are more or less alike; hence, the regularization order $s=0$ is omitted in the coming presentation. The numerical solution $u_{5 \%}^{-1}\left(R_{i}, \theta\right)$ looks more accurate than $u_{5 \%}^{0.5}\left(R_{i}, \theta\right)$. However the normal derivative $\partial_{r} u_{5 \%}^{-1}\left(R_{i}, \theta\right)$ contains higher oscillation than $\partial_{r} u_{5 \%}^{0.5}\left(R_{i}, \theta\right)$ as one can see in Fig. 3. On the other hand, in contrast to the case $s=-1$, the numerical solution $u_{5 \%}^{1}\left(R_{i}, \theta\right)$ and its normal derivative $\partial_{r} u_{5 \%}^{1}\left(R_{i}, \theta\right)$ is flat. The authors observed that regardless of the relative noise level $\sigma$, the numerical solution $u_{\sigma}^{-1}\left(R_{i}, \theta\right)$ looked more "aggressive" and $u_{\sigma}^{1}\left(R_{i}, \theta\right)$ more "conservative". Therefore, we should consider a strat-

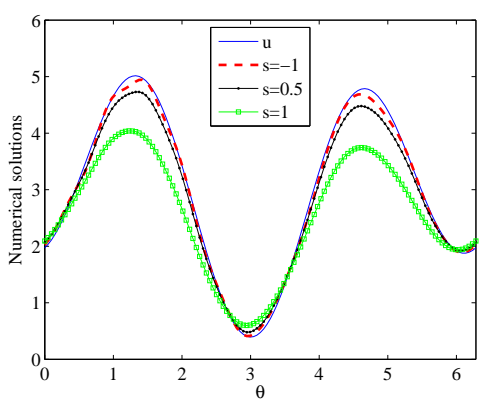

Figure 2: Example 3.1: The exact solution $u\left(R_{i}, \theta\right)$ and numerical solutions, $u_{5 \%}^{-1}\left(R_{i}, \theta\right)$, $u_{5 \%}^{0.5}\left(R_{i}, \theta\right)$ and $u_{5 \%}^{1}\left(R_{i}, \theta\right)$.

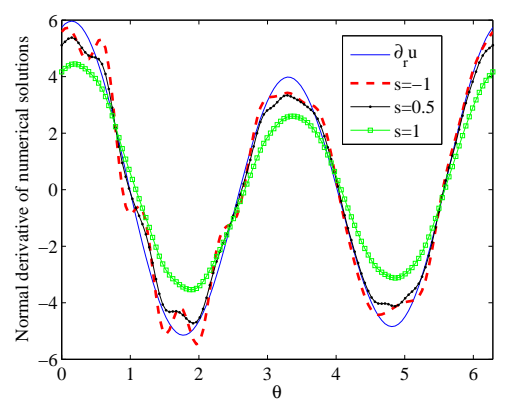

Figure 3: Example 3.1: The exact solution $\partial_{r} u\left(R_{i}, \theta\right)$ and the normal derivative of the numerical solutions, $\partial_{r} u_{5 \%}^{-1}\left(R_{i}, \theta\right), \partial_{r} u_{5 \%}^{0.5}\left(R_{i}, \theta\right)$ and $\partial_{r} u_{5 \%}^{1}\left(R_{i}, \theta\right)$. 
egy to pick the regularization order $s$ according to given Cauchy data in order to get an accurate numerical solution. We leave this to a future work.

\section{Example 3.2.}

$$
u(r, \theta)=5-\log \left((r \cos \theta-0.6)^{2}+(r \sin \theta)^{2}\right) .
$$

Note that, in this example, the Fourier expansion of $u$ is an infinite series. This makes Example 3.2 more difficult to solve accurately than Example 3.1.

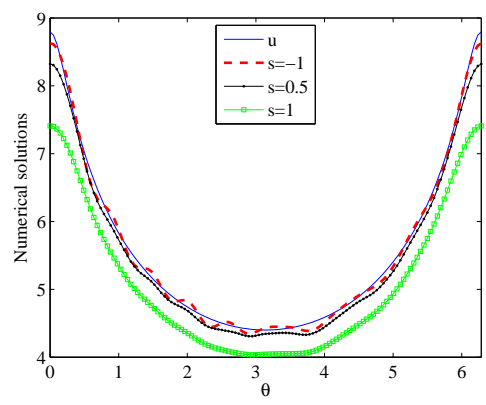

Figure 4: Example 3.2: The exact solution $u\left(R_{i}, \theta\right)$ and numerical solutions, $u_{5 \%}^{-1}\left(R_{i}, \theta\right)$, $u_{5 \%}^{0.5}\left(R_{i}, \theta\right)$ and $u_{5 \%}^{1}\left(R_{i}, \theta\right)$.

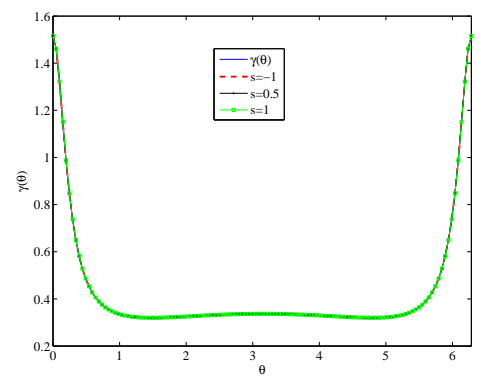

with noise free Cauchy data

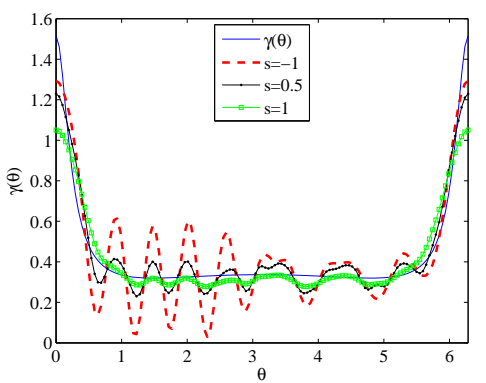

with $5 \%$ noise

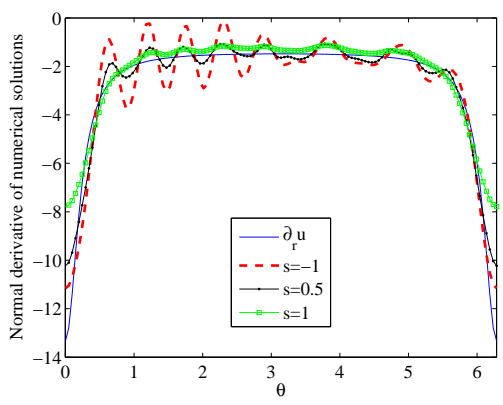

Figure 5: Example 3.2: The exact solution $\partial_{r} u\left(R_{i}, \theta\right)$ and the normal derivative of the numerical solutions, $\partial_{r} u_{5 \%}^{-1}\left(R_{i}, \theta\right), \partial_{r} u_{5 \%}^{0.5}\left(R_{i}, \theta\right)$ and $\partial_{r} u_{5 \%}^{1}\left(R_{i}, \theta\right)$.

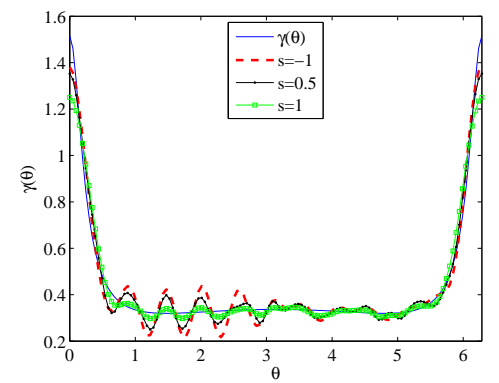

with $1 \%$ noise

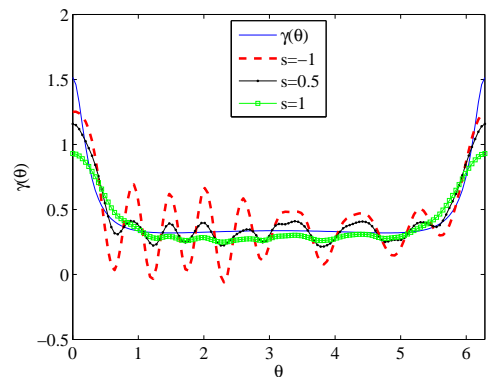

with $10 \%$ noise

Figure 6: Example 3.2: Numerical reconstruction of the coefficient of energy exchange $\gamma_{0 \%}^{s}(\theta), s \in\{-1,0.5,1\}$. 


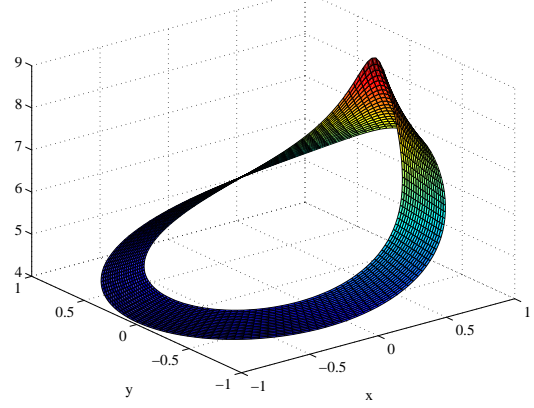

$u_{\sigma}^{s}, \sigma=0 \%$
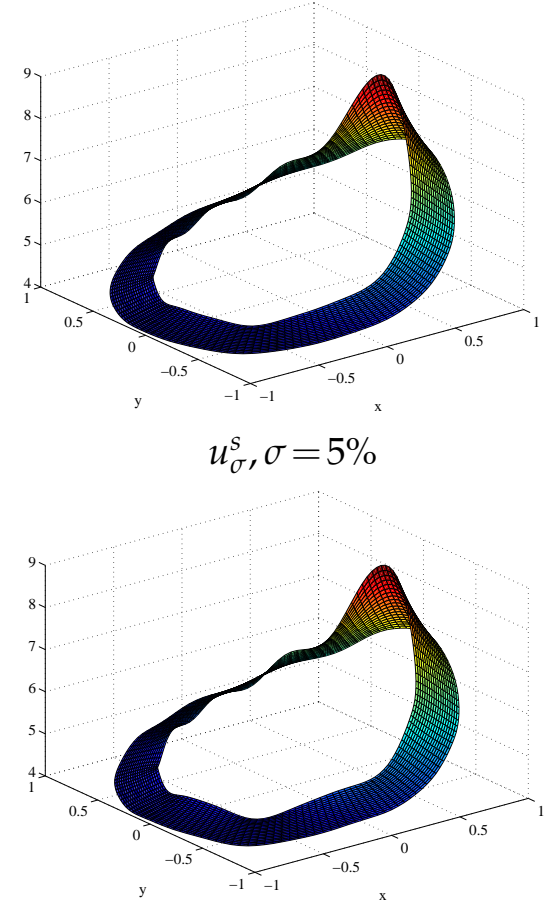

$u_{\sigma}^{s}, \sigma=10 \%$

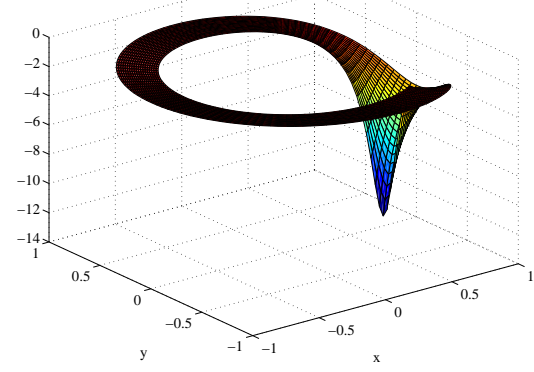

$\partial_{r} u_{\sigma}^{s}, \sigma=0 \%$

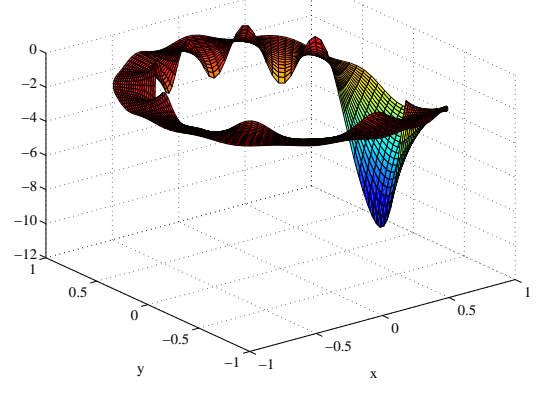

$\partial_{r} u_{\sigma}^{s}, \sigma=5 \%$

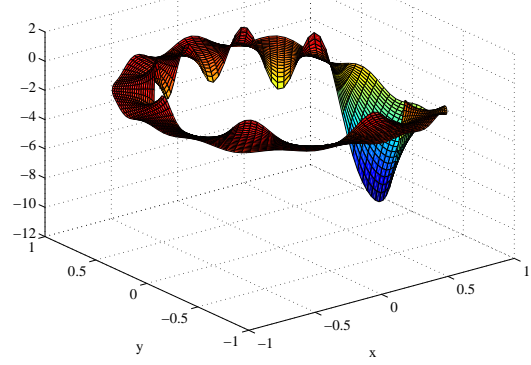

$\partial_{r} u_{\sigma}^{s}, \sigma=10 \%$

Figure 7: Example 3.2: Numerical solutions and their $r$-derivatives using regularization order $s=-1$ under different relative noise levels $\sigma$.

The graph of the resulting relative error $E(\sigma, s)$ shows the same trend as that of Example 3.1 (see Fig. 1) and is omitted.

We plot three numerical solutions on the inner boundary $u_{5 \%}^{-1}\left(R_{i}, \theta\right), u_{5 \%}^{0.5}\left(R_{i}, \theta\right)$, $u_{5 \%}^{1}\left(R_{i}, \theta\right)$ and the exact solution $u\left(R_{i}, \theta\right)$ in Fig. 4 and their normal derivative in Fig. 5. As one have seen in Example 3.1, the numerical solution for $s=-1$ is more aggressive and $s=1$ is more conservative.

From Fig. 4 and Fig. 5, it may appear that using regularization order $s=1$ results less superior approximations of $u\left(R_{i}, \cdot\right)$ and $\partial_{r} u\left(R_{i}, \cdot\right)$ than the other two choices. Before end- 

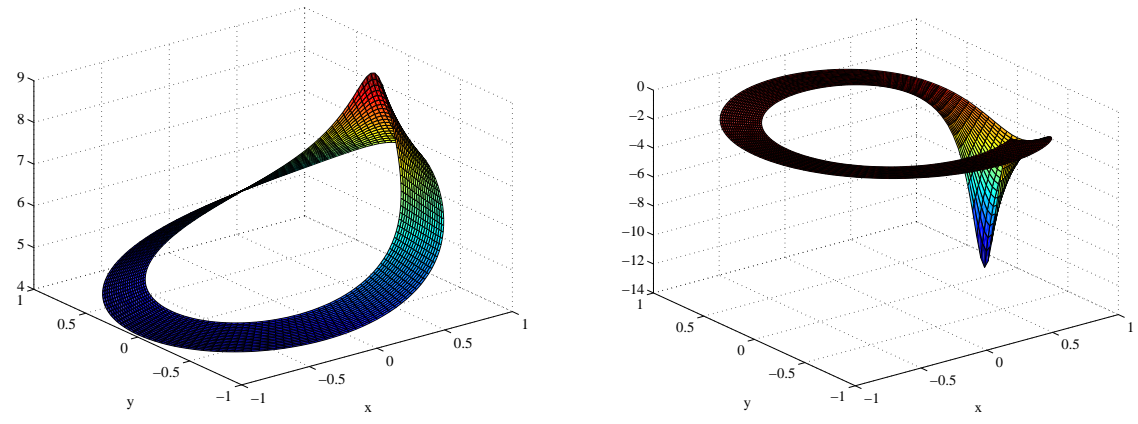

$$
u_{\sigma}^{s}, \sigma=0 \%
$$

$$
\partial_{r} u_{\sigma}^{s}, \sigma=0 \%
$$
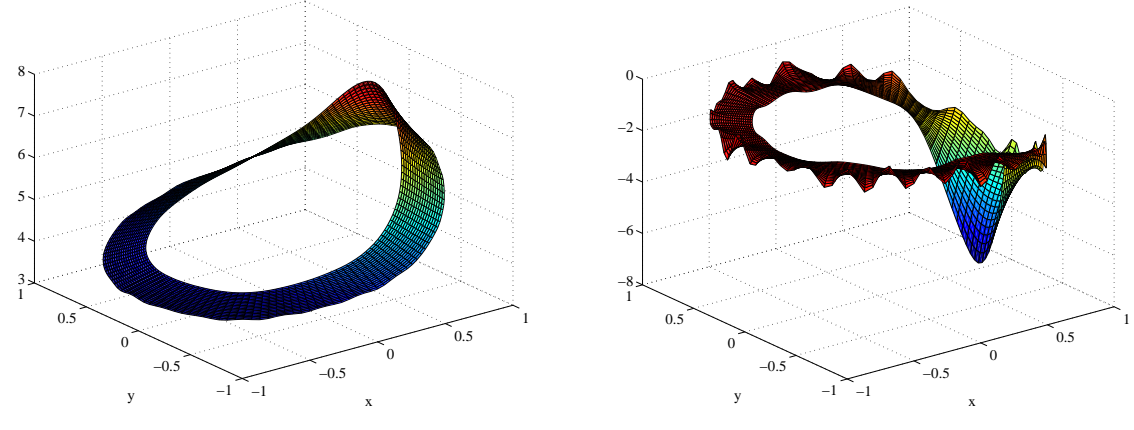

$$
u_{\sigma}^{s}, \sigma=5 \%
$$

$$
\partial_{r} u_{\sigma}^{s}, \sigma=5 \%
$$
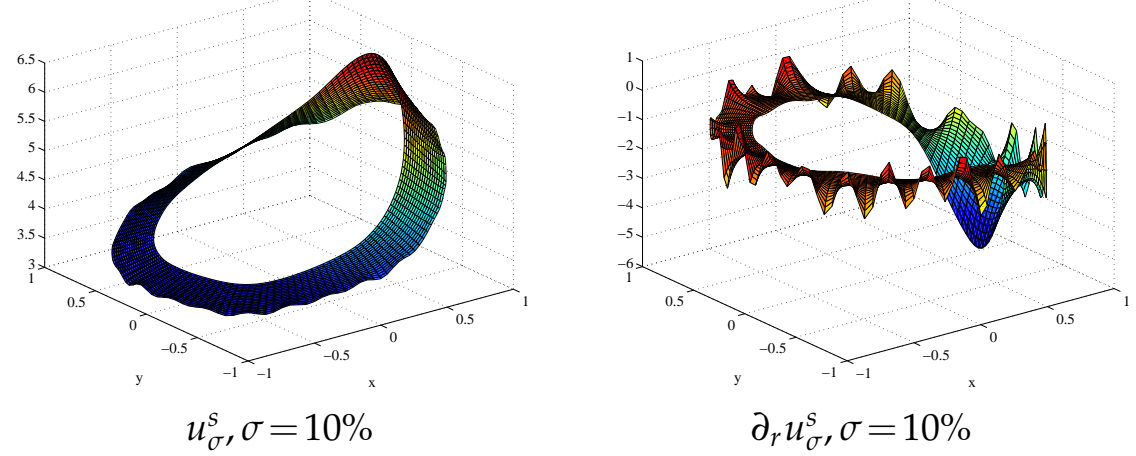

Figure 8: Example 3.2: Numerical solutions and their $r$-derivatives using regularization order $s=1$ under different relative noise levels $\sigma$.

ing our discussion, we shift our focus to the important physical quantity-the coefficient of energy exchange $\gamma$. Approximation to $\gamma$ can be immediately obtained via:

$$
\gamma_{\sigma}^{s}(\theta):=-\frac{\partial_{r} u_{\sigma}^{s}\left(R_{i}, \theta\right)}{u_{\sigma}^{s}\left(R_{i}, \theta\right)}
$$

For $s \in\{-1,0.5,1\}$, the numerical reconstructions of the energy exchanges are shown in Fig. 6(a) and in Fig. 6(c), respectively, for the noise free and noisy Cauchy data with relative noise level $\sigma \in\{0 \%, 1 \%, 5 \%, 10 \%\}$. For the noise free case (e.g., $\sigma=0$ in Fig. 6(a)), all 
tested $s$ result in perfect reconstructions of the coefficient $\gamma$. However, when the Cauchy data is contaminated by noise, Fig. 6(b) to Fig. 6(d) show that the seemingly bad choice $s=1$ gives the best approximation to $\gamma$ in terms of the overall shape. Reconstructed $\gamma_{\sigma}^{1}$ is less oscillatory, even under $10 \%$ of relative noise, than the others (smaller) $s$ and is still able to capture the "peak" around $\theta=0$. To better demonstrate the effect of $s$, Fig. 7 and Fig. 8 demonstrate the numerical solutions and their $r$-derivatives using regularization order $s \in\{-1,1\}$, respectively, under relative noise levels $\sigma=\{0 \%, 5 \%, 10 \%\}$. Plots for $s=0.5$ are similar to that of $s=-1$ and are omitted. Moreover, we remark that the Cauchy data for the same $\sigma$ are identical in both figures. One can see that the numerical solutions of $s=1$ appears to be more oscillatory near the outer boundary; on the inner boundary, the $r$-derivatives in Fig. 8 are comparatively smoother than those in Fig. 7.

\section{Conclusions}

We prove a three-line inequality for the Cauchy problem of Laplace equation in annulus domain. By this inequality, we show that the Cauchy solution depends continuously on the Cauchy data posed on the outer boundary if we assume that the solution trace on the inner boundary are bounded. These results naturally lead us to an energy regularization method; the regularized solution can be sought within a (Cauchy dependent) subspace of $H^{s+1 / 2}(\Omega)$. Convergence analysis for the energy regularization method is relatively simple. Later, we build a connection between our method and the commonly used Tikhonov regularization. It is shown what the energy regularization is in fact equivalent to a Tikhonov regularization.

A numerical procedure based on the method of fundamental solution is proposed. Instead of strong form collocation, we couple the energy regularization on the Fourier space. For noise free data, numerical results show machine accuracy on the reconstructions of Dirichlet and Neumann inner boundary data for all tested regularization order s. For contaminated Cauchy data, small regularization order (e.g., $s=-1$ ) is better if the Dirichlet and Neumann boundary data are desired. On the order hand, when one is interested in the coefficient of energy exchange, large regularization order (e.g., $s=1$ ) is a better choice. We tested up to $10 \%$ of relative noise, the overall shape of the coefficient of energy exchange can still be well approximated.

A question to be answered is the strategy of pick the "noise level" that is used to define the subspace in which lies the numerical solution. We leave this to our future research.

\section{Acknowledgments}

This project was supported by a CERG Grant of Hong Kong Research Grant Council, a FRG grant of Hong Kong Baptist University, and was partially supported by the NSFC Project No. 19971116. 


\section{References}

[1] G. Inglese, An inverse problem in corrosion detection, Inverse. Probl., 13 (4) (1997), 977-994.

[2] G. Buttazzo, and R. V. Kohn, Reinforcement by a thin layer with oscillating thickness, Appl. Math. Opt., 16 (3) (1987), 247-261.

[3] G. Inglese, and F. Santosa, An Inverse Problem in Corrosion Detection, No. 75, Pubblicazioni dellIstituto di Analisi Globale ed Applicazioni del CNR, Firenze, 1995.

[4] M. H. Protter, and H. F. Weinberger, Maximum Principles in Differential Equations, Springer-Verlag, New York, 1984, corrected reprint of the 1967 original.

[5] G. Alessandrini, Stable determination of a crack from boundary measurements, Proc. Roy. Soc. Edinburgh. Sec. A., 123(3) (1993), 497-516.

[6] N. M. Al-Najem, A. M. Osman, M. M. El-Refaee, and K. M. Khanafer, Two dimensional steady-state inverse heat conduction problems, Int. Commun. Heat. Mass. Trans., 25(4) (1998), 541-550.

[7] P. Colli Franzone, and E. Magenes, On the inverse potential problem of electrocardiology, Calcolo., 16(4) (1979), 459-538.

[8] H. Han, The finite element method in the family of improperly posed problems, Math. Comput., 38(157) (1982), 55-65.

[9] H. Han, and H.-J. Reinhardt, Some stability estimates for Cauchy problems for elliptic equations, J. Inverse. Ill-Pose. Probl., 5(5) (1997), 437-454.

[10] R. S. Falk, and P. B. Monk, Logarithmic convexity for discrete harmonic functions and the approximation of the Cauchy problem for Poisson's equation, Math. Comput., 47(175) (1986), 135-149.

[11] D. D. Ang, N. H. Nghia, and N. C. Tam, Regularized solutions of Cauchy problem for the laplace equation in an irregular layer: a three dimensional case, Acta. Math. Vietnamica., 23 (1998), 65-74.

[12] F. Berntsson, and L. Eldén, Numerical solution of a Cauchy problem for the Laplace equation, Inverse. Probl., 17(4) (2001), 839-853.

[13] H.-J. Reinhardt, H. Han, and D. N. Háo, Stability and regularization of a discrete approximation to the Cauchy problem for Laplace's equation, SIAM J. Numer. Anal., 36(3) (1999), 890-905.

[14] J. Cheng, Y. C. Hon, T. Wei, and M. Yamamoto, Numerical computation of a Cauchy problem for Laplace's equation, ZAMM Z. Angew. Math. Mech., 81(10) (2001), 665-674.

[15] Y. C. Hon, and T. Wei, Backus-Gilbert algorithm for the Cauchy problem of the Laplace equation, Inverse. Probl., 17(2) (2001), 261-271.

[16] T. Wei, Y. C. Hon, and L. Ling, Method of fundamental solutions with regularization techniques for Cauchy problems of elliptic operators, Eng. Anal. Bound. Elem., 31(4) (2007), 373-385.

[17] T. Takeuchi, and M. Yamamoto, Tikhonov regularization by a reproducing kernel Hilbert space for the Cauchy problem for an elliptic equation, SIAM J. Sci. Comput., 31(1) (2008), 112-142.

[18] A. Leitão, An iterative method for solving elliptic Cauchy problems, Numer. Funct. Anal. Opt., 21(5-6) (2000), 715-742.

[19] T. Wei, H. H. Qin, and R. Shi, Numerical solution of an inverse 2D Cauchy problem connected with the Helmholtz equation, Inverse. Probl., 24(3) (2008), 035003.

[20] X. Yang, M. Choulli, and J. Cheng, An iterative BEM for the inverse problem of detecting corrosion in a pipe, Num. Math., J. China. Univ., 14(3) (2005), 252-266. 
[21] H. Han, M. Yan, and C. Wu, An energy regularization method for the backward diffusion problem and its applications to image deblurring, Commun. Comput. Phys., 4(1) (2008), 177-197.

[22] J. Baumeister, Stable Solution of Inverse Problems, Advanced Lectures in Mathematics, Friedr. Vieweg \& Sohn, Braunschweig, 1987.

[23] H. W. Engl, M. Hanke, and A. Neubauer, Regularization of Inverse Problems, Vol. 375 of Mathematics and its Applications, Kluwer Academic Publishers Group, Dordrecht, 1996.

[24] C. W. Groetsch, The Theory of Tikhonov Regularization for Fredholm Equations of the First Kind, Vol. 105 of Research Notes in Mathematics, Pitman (Advanced Publishing Program), Boston, MA, 1984.

[25] C. W. Groetsch, Inverse Problems in the Mathematical Sciences, Vieweg Mathematics for Scientists and Engineers, Friedr. Vieweg \& Sohn, Braunschweig, 1993.

[26] B. Hofmann, On the Generalized Discrepancy Principle in Regularization, Vol. 48, Wissenschaftliche Informationen, 1984.

[27] B. Hofmann, Regularization for Applied Inverse and Ill-Posed Problems, Vol. 85 TeubnerTexte zur Mathematik [Teubner Texts in Mathematics], 1986.

[28] V. V. Vasin, Some tendencies in the Tikhonov regularization of ill-posed problems, J. Inverse. Ill-Pose. Probl., 14(8) (2006), 813-840.

[29] K. Ito, and K. Kunisch, On the choice of the regularization parameter in nonlinear inverse problems, SIAM J. Opt., 2(3) (1992), 376-404.

[30] K. Kunisch, and J. Zou, Iterative choices of regularization parameters in linear inverse problems, Inverse. Probl., 14(5) (1998), 1247-1264.

[31] J. Xie, and J. Zou, An improved model function method for choosing regularization parameters in linear inverse problems, Inverse. Probl., 18(3) (2002), 631-643.

[32] C. M. Fan, C. S. Chen, and J. Monroe, The method of fundamental solutions for solving convection-diffusion equations with variable coefficients, Adv. Appl. Math. Mech., 1(2) (2009), 215-230.

[33] A. Karageorghis, and D. Lesnic, The method of fundamental solutions for steady-state heat conduction in nonlinear materials, Commun. Comput. Phys., 4(4) (2008), 911-928.

[34] Y. Hon, and T. Wei, A fundamental solution method for inverse heat conduction problem, Eng. Anal. Bound. Elem., 28(5) (2004), 489-495.

[35] Y. Hon, and T. Wei, The method of fundamental solution for solving multidimensional inverse heat conduction problems, CMES Comput. Model. Eng. Sci., 7(2) (2005), 119-132.

[36] L. Ling, and T. Takeuchi, Point sources identification problems for heat equations, Commun. Comput. Phys., 5(5) (2009), 897-913.

[37] L. Ling, M. Yamamoto, Y. C. Hon, and T. Takeuchi, Identification of source locations in twodimensional heat equations, Inverse. Probl., 22(4) (2006), 1289-1305. 